\title{
Summary of SiC Research for Transportation Applications at ORNL
}

\author{
Madhu Chinthavali, a , Burak Ozpineci ${ }^{1, b}$, Leon M. Tolbert ${ }^{2, c}$, Hui Zhang ${ }^{2, d}$ \\ ${ }^{1}$ Oak Ridge National Laboratory, Knoxville, TN, USA - 37932 \\ ${ }^{2}$ University of Tennessee, Knoxville, TN, USA -37996 \\ achinthavalim@ornl.gov, bburak@ieee.org, ctolbert@utk.edu, 'hzhang5@utk.edu
}

Keywords: SiC, high temperature, transportation applications.

\begin{abstract}
The theoretical advantages that Silicon Carbide ( $\mathrm{SiC}$ ) material offers are being realized by using prototype or experimental devices in many different applications ranging from medium voltage to high voltage. The main advantages of using $\mathrm{SiC}$ based devices are reduced thermal management requirements and smaller passive components which result in higher power density. An overview of the $\mathrm{SiC}$ research effort for transportation applications at Oak Ridge National Laboratory (ORNL) is presented in this paper.
\end{abstract}

\section{Introduction}

In pursuit of mass production of hybrid electric vehicles, the automotive research industry has set goals such as reducing the size and weight of the power electronics and cooling systems and increasing their efficiency. U. S Departemnt of Energy goals for hybrid electric vehicles in 2020 are for power electronics to be atleast $14.1 \mathrm{~kW} / \mathrm{Kg}$ and $13.4 \mathrm{~kW} / \mathrm{L}$, efficiency greater than $98 \%$, and cost less than $\$ 3.3 / \mathrm{kW}$. SiC devices are capable of operating at higher voltages, higher frequencies, and higher junction temperatures, which result in significant reduction in weight and size of the power converter and an increase in efficiency. The objectives of research efforts on $\mathrm{SiC}$ based device applications at ORNL are

- Assessing the impact of replacing silicon (Si) power devices in transportation applications with wide-bandgap (WBG) semiconductors, especially silicon carbide ( $\mathrm{SiC}$ ).

- Developing device models for drive train system simulation studies, and analyze the impact of $\mathrm{SiC}$ devices on the system performance.

- Building SiC-based prototype converters to validate the performance of SiC devices.

- Building high temperature packages for $\mathrm{SiC}$ power devices to operate at $200^{\circ} \mathrm{C}$ ambient.

\section{Device Characterization and Modeling}

The static and dynamic characteristics of the devices are obtained experimentally. The experimental data is analyzed to extract device parameters to develop behavioral models. The behavioral models are integrated into a system level model used to study their impact on the system performance.

\section{Static Characteristics}

$\mathrm{I}-\mathrm{V}$ characteristics of the $600 \mathrm{~V} / 75 \mathrm{~A} \mathrm{SiC} \mathrm{Schottky} \mathrm{diode} \mathrm{were} \mathrm{obtained} \mathrm{at} \mathrm{different} \mathrm{temperatures} \mathrm{in}$ the $-50^{\circ} \mathrm{C}$ to $175^{\circ} \mathrm{C}$ temperature range (Fig. 1). Considering the piece-wise linear (PWL) model of a diode, which includes a de voltage drop, $\mathrm{V}_{\mathrm{D}}$, and a series resistor, $\mathrm{R}_{\mathrm{D}}$; the diode $\mathrm{I}-\mathrm{V}$ curves can be approximated with the following equation [1]:

$$
V_{d}=V_{D}+R_{D} \cdot I_{d}
$$

Where $V_{d}$ and $I_{d}$ are the diode forward voltage and current, and $V_{D}$ and $R_{D}$ are the diode PWL model

Prepared by the Oak Ridge National Laboratory, Oak Ridge, Tennessee 37831, managed by UT-Battelle for the U.S. Department of Energy under contract DE-AC05-00OR22725.

The submitted manuscript has been authored by a contractor of the U.S. Government under Contract No. DE-AC05-00OR22725. Accordingly, the U.S. Government retains a non-exclusive, royalty-free license to publish from the contribution, or allow others to do so, for U.S. Government purposes. 
parameters.

$V_{D}$ decreases with temperature and $R_{D}$ increases with temperature. The increase in $R_{D}$ is an effect of the positive temperature coefficient the SiC Schottky diodes have, which enables easy paralleing of these devices. Equations below show the temperature dependence of the SiC Schottky diode PWL model parameters:

$V_{D}=-0.001 \cdot T+0.94$ (Volts)

$R_{D}=8.9 \times 10^{-5} \cdot T+0.013(\mathrm{Ohms})$

Where $T$ is the temperature in ${ }^{\circ} \mathrm{C}$.

\section{Dynamic Characteristics}

The SiC Schottky diode was also tested in a chopper circuit to observe its dynamic characteristics. A

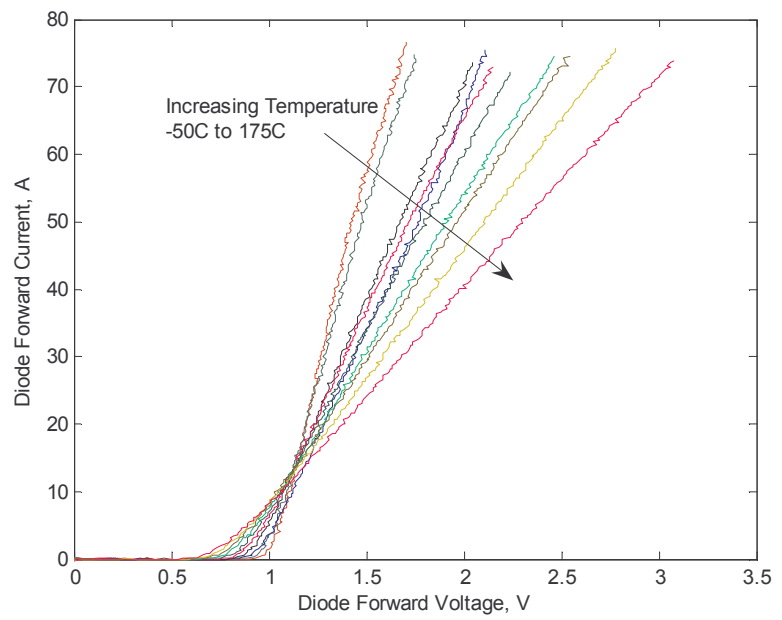

Fig.1: Experimental i-v curves of a $75 \mathrm{~A} \mathrm{SiC}$ Schottky diode.

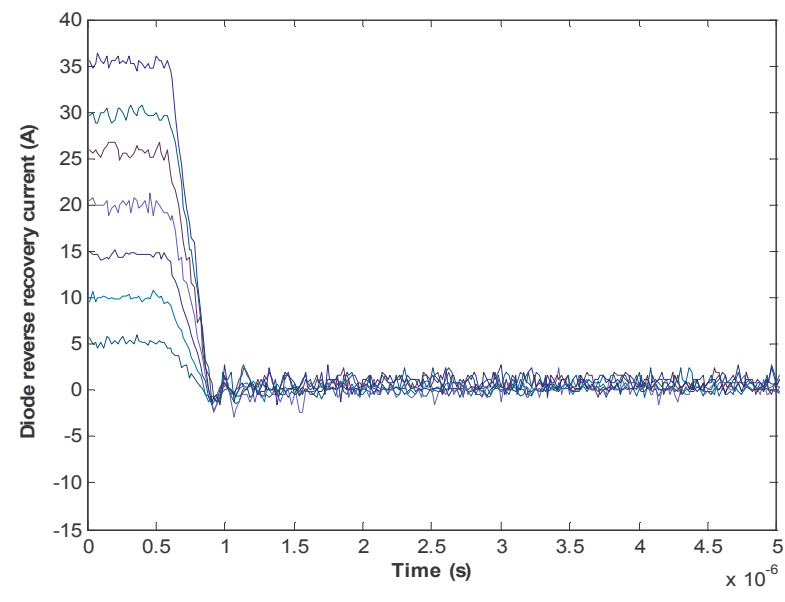

Fig. 2: Reverse recovery waveforms of a SiC Schottky diode for different forward currents.

double pulse circuit was used to obtain the turn-on and turn-off energy losses. The reverse recovery current waveforms obtained for different forward currents at room temperature are shown in Fig. 2. As seen in this figure, the reverse recovery current does not change with forward current. Note that theoretically, Schottky diodes do not display reverse recovery phenomenon because they are majority carrier devices and do not have stored charge. The switching losses were also obtained at different temperatures. The losses do not change much with temperature, and hence the losses can be modeled as given below.

$$
E_{s w}=-6.5 \times 10^{-9} \cdot I+3.2 \times 10^{-7} \quad(\text { Joules })
$$

The behavioral device models are used to develop inverter loss models to study the system level benefits. Three different inverter models were simulated for several operating conditions and some of the results are discussed below. The efficiency versus output power plots for several operating conditions comparing the inverters is shown are Fig. 3. The percentage loss reduction was calculated comparing the power loss difference between the hybrid inverter and the all-SiC inverter with respect to the all-Si inverter. The hybrid inverter and all-SiC efficiencies are higher than the all-Si inverter for all operating conditions. The percentage loss reduction was calculated comparing the power loss difference between the hybrid inverter and the all-SiC inverter with respect to the all-Si inverter.

$$
\% \text { loss reduction }=\frac{P_{\text {loss }}^{A l l-S i}-P_{\text {loss }}^{A l l-S i C}}{P_{\text {loss }}^{A l l-S i}} \times 100
$$




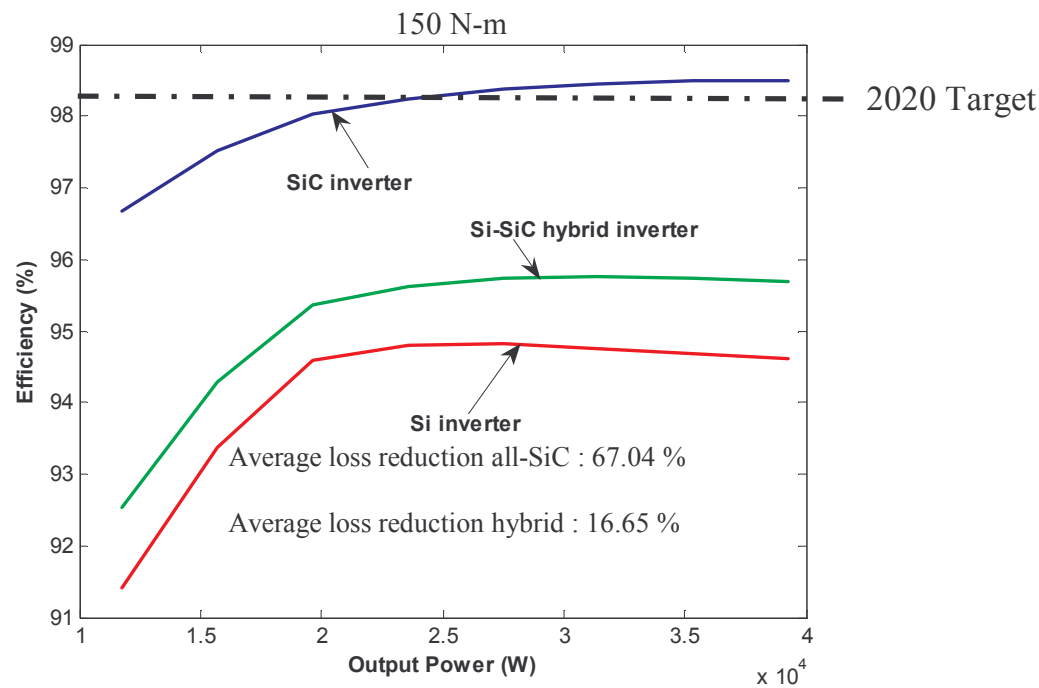

Fig. 3: Efficiency versus output power of three different inverters at $150 \mathrm{~N}-\mathrm{m}$ load.

\section{55 kW Hybrid Inverter}

SiC Schottky diodes have been proven to have better performance characteristics when compared to similar Si pn diodes [1, 2], especially with respect to their switching characteristics as they have negligible reverse-recovery losses. The superior switching performance of the diodes impacts the main power switches by reducing the stress on them and thus improving system performance. SiC Schottky diodes are already commercially available at current ratings up to $50 \mathrm{~A}$. These diodes are being used in niche applications such as power factor correction circuits. It is expected that the first impact of SiC devices on inverters will be as a result of SiC Schottky diodes replacing the Si pn diodes.

ORNL collaborated with Cree and Semikron to build a hybrid 55 kW (Si IGBT-SiC Schottky diode) inverter by replacing the Si pn diodes in Semikron's automotive inverter with Cree's SiC Schottky diodes [3]. A comparison of performance between the all-Si and the hybrid inverter is shown in Fig. 4 and Fig. 5. The hybrid inverter efficiencies are higher than the all-Si inverter for all operating conditions. The results show up to $33.6 \%$ reduction in the losses when everything is kept the same and SiC Schottky diodes are used instead of Si pn diodes.

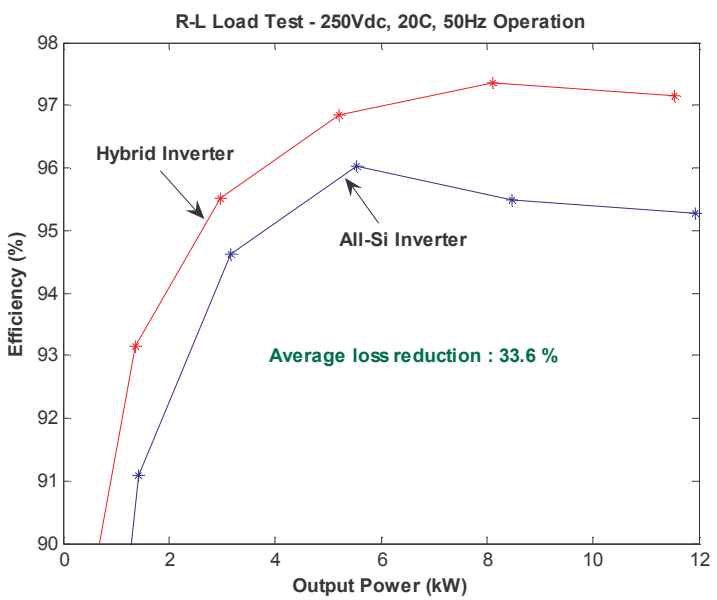

Fig. 4: R-L load test efficiency curves for one of the load conditions.

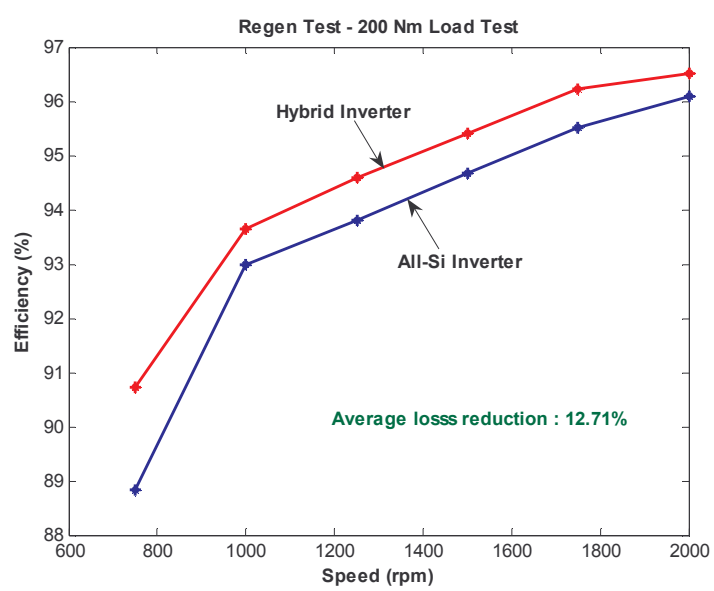

Fig. 5: Dynamometer test-regeneration mode efficiency plots at $70^{\circ} \mathrm{C}$. 


\section{High Temperature Packaging}

One of the most important characteristics of $\mathrm{SiC}$ power devices is that they can operate at much higher temperatures $\left(>300^{\circ} \mathrm{C}\right)$ than Si power devices. Presently, SiC devices use Si device packages that limit the operation temperature $\left(\sim 150^{\circ} \mathrm{C}\right)$. ORNL collaborated with Univ. of Arkansas to build several high temperature packages to demonstrate high temperature packages that can operate at $200^{\circ} \mathrm{C}$ ambient [4].

A single die $600 \mathrm{~V} / 75 \mathrm{~A}$ SiC Schottky diode was packaged in a TO-220 thru-hole style package shown in Fig. 6. The package was tested at different current levels at $200^{\circ} \mathrm{C}$ ambient temperature without a heat sink. The case temperature of the diode was measured using a thermocouple. The diode operated at $361^{\circ} \mathrm{C}$ at $20 \mathrm{~A}$ for over an hour without a failure. However at $25 \mathrm{~A}$ the diode operated for a short time and it failed short. This is a significant milestone in terms of operating temperature of the device which clearly demonstrates the high temperature operation capability of SiC devices.
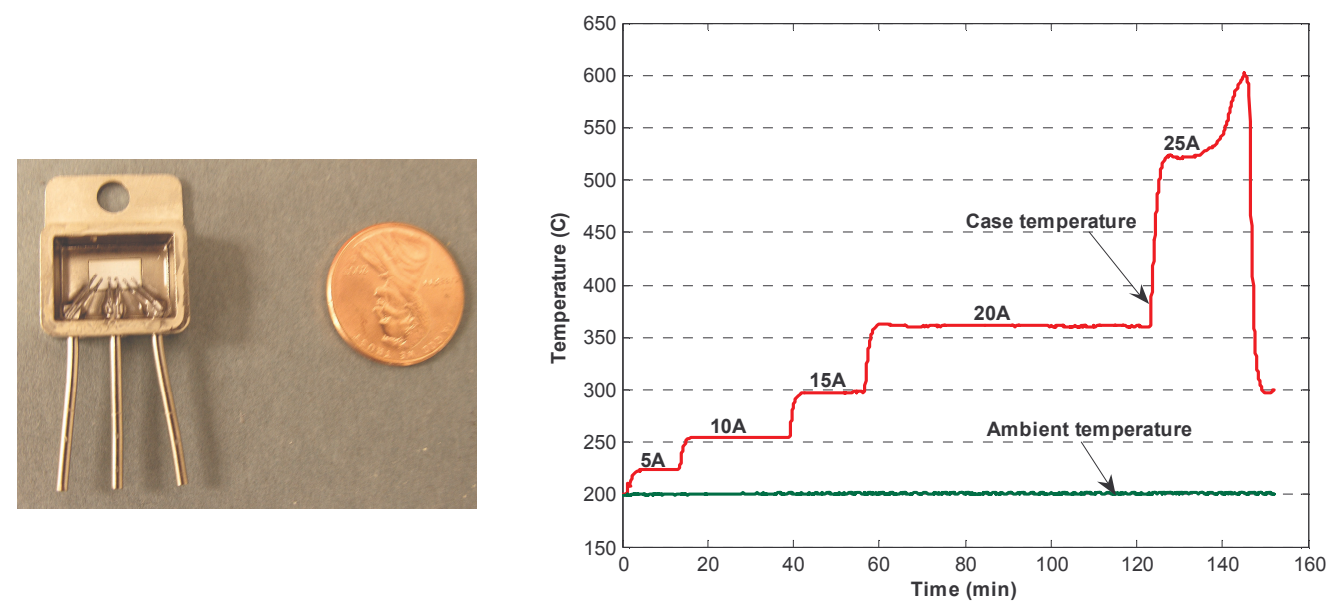

Fig.6 : TO-220 through -hole high temperature package of 75 A SiC Schottky diode and test results.

\section{Conclusions}

These studies on $\mathrm{SiC}$ based power electronics revealed some important results, which were discussed with the device manufacturers to further improve the design and fabrication. The impact of SiC devices on system level performance parameters compared to Si devices makes SiC material a better choice for transportation applications. The $\mathrm{SiC}$ research program at ORNL has been improving with continued effort of keeping abreast with the maturing SiC technology. It is important to follow the present developments in $\mathrm{SiC}$ power device technology closely because the pace of improvement has increased considerably.

\section{References}

[1] B. Ozpineci, M. Chinthavali, L. M. Tolbert, "Enhancing Power Electronic Devices with Wide Bandgap Semiconductors," Advanced Workshop on 'Frontiers of Electronics' (WOFE'04), Aruba, December 17-22, 2004.

[2] B. Ozpineci, L. M. Tolbert, "Characterization of SiC Schottky Diodes at Different Temperatures," IEEE Power Electronics Letters, vol. 1, no. 2, June 2003, pp. 54-57.

[3] B. Ozpineci, M. Chinthavali, A. Kashyap, L. M. Tolbert, A. Mantooth, "A 55 kW Three-Phase Inverter With Si IGBTs and SiC Schottky Diodes,” IEEE Applied Power Electronics Conference, March 19-23, 2006, Dallas, Texas, pp.448-454.

[3] S. A. Rogers, "FreedomCAR and Vehicle Technologies Annual Progress Report for the Advanced Power Electronics Technology Area," December 2006. 